\title{
Quality Assessment with HPLC in Released Varieties of Tetraploid (Triticum durum Desf.) Wheat from Ethiopia and Spain
}

\author{
F. Hailu ${ }^{*}$, M. Labuschagne ${ }^{2}$, A. Van Biljon ${ }^{2}$, H. Persson Hovmalm ${ }^{3}$ and E. Johansson ${ }^{3}$ \\ ${ }^{1}$ Department of Biology, College of Natural Science, Wollo University, \\ Personal P. O. Box-1022, Dessie, Ethiopia \\ ${ }^{2}$ Department of Plant Sciences, University of the Free State, \\ P. O. Box: 339, Bloemfontein 9300, South Africa \\ ${ }^{3}$ Department of Plant Breeding, Swedish University of Agricultural Sciences, \\ P. O. Box-101, SE-230 53 Alnarp, Sweden \\ (Received 5 May 2015; Accepted 29 June 2016; \\ Communicated by F. Békés)
}

\begin{abstract}
Quality of durum wheat is of importance for pasta production. Our aim was to evaluate the quality of released durum wheat at Ethiopia by the use of pheno-quality traits as well as composition and amount and size distribution of proteins; these attributes were also compared with the same ones for Spanish released wheat grown in the same environment. Large variation was obtained among the released durum wheat for all parameters investigated. The evaluation of protein parameters indicated the varieties Klinto, LD-357, Tob-66 and Ude to have suitable protein composition for pasta production, while Tob-66 showed high protein concentration and Tob-66, LD-357 and Yerer showed high gluten strength. The most promising Ethiopian variety for pasta production as to the results from the present study was Tob-66. Also, LD-357 showed promising protein characteristics, although the kernels were white, which is not desired for pasta production. Some of the evaluated varieties might be of interest for production of local leavened bread although the quality is not good enough for pasta production.
\end{abstract}

Keywords: durum wheat, Ethiopia, Spain, SE-HPLC, released varieties and RP-HPLC

\section{Introduction}

Durum wheat (Triticum turgidum L. subsp. durum) is one of the tetraploid (AABB, $2 n=4 x=28$ ) wheat species of commercial importance and it has specific quality attributes making it suitable for pasta production (Bechere et al. 2002). Therefore, durum wheat is widely cultivated at present although it is not grown to the same extent as common hexaploid wheat (Peña et al. 2002). Most work as related to baking performance and its background, has been carried out in hexaploid wheat (Johansson et al. 2013), although substantial variation has been seen for gluten strength and baking performance also in durum wheat (Boggini and Pogna 1989; Rharrabti et al. 2003). The technological proper-

*Corresponding author; E-mail: markhmets@yahoo.com 
ties of wheat flour are strongly dependent on wheat genotype and growing conditions, and are mainly determined by composition, structure and quantity of gluten proteins (Wieser et al. 1998; Gulia and Khatkar 2015). Protein polymerization during dough formation and baking is among the most important parameters determining bread-making quality (Johansson et al. 2013). The ability of the wheat proteins to polymerise can be measured using Size Exclusion-High Performance Liquid Chromatography (SE-HPLC) in the grain (Johansson et al. 2005, 2008) and in the dough (Hussain et al. 2012). Besides SE-HPLC, also reversed-phase (RP)-HPLC has frequently been used to relate the quantity of gluten protein fractions to flour properties (Gupta and Macritchie 1994; El Haddad et al. 1995; Gupta et al. 1995, 1996; Ciaffi et al. 1996; Fu and Sapirstein 1996; Wieser et al. 1998). The SE-HPLC has been used in some studies to evaluate the protein polymerisation in durum wheat during pasta production (Liu et al. 1996). However, to our knowledge, no study is available that has evaluated genotypic variation in protein composition and amount and size distribution of polymeric protein in durum wheat using SE- and RPHPLC. Furthermore no study has evaluated relationships between the mentioned protein parameters and quality in durum wheat.

The aim of this study was to i) evaluate variation in amount and size distribution of polymeric protein as well as protein composition determined by SE- and RP-HPLC and SDS-PAGE in released varieties of durum wheat both at Ethiopia and Spain, ii) investigate whether the mentioned protein parameters differ between Ethiopian released varieties and some Spanish released durum wheat varieties grown in the same environment, and iii) determine whether significant correlations exist between the mentioned protein parameters and different quality assessment parameters normally used to assess the quality of durum wheat.

\section{Materials and Methods}

\section{Plant material}

Ten released varieties of tetraploid wheat were obtained from the Debre Zeit Agricultural Research Centre, Ethiopia and additional five released varieties were obtained from Spain. The sample was taken randomly as a matter of chance from Spain and Ethiopia. All the fifteen varieties were grown in Dessie Zuria district at a farmer's piece of land (Table S1*).

\section{Experimental design and pheno-quality evaluation}

The wheat varieties were sown in the 2009 main cropping season applying random complete block design with two replications. The plot size was $2.5 \mathrm{~m}^{2}$ consisting of six rows, $2.5 \mathrm{~m}$ long and spaced apart by $20 \mathrm{~cm}$. Standard management practices were exercised. The pheno-quality traits were characterized according to Hailu et al. (2010).

*Further details about the Electronic Supplementary Material (ESM) can be found at the end of the article. 


\section{Analysis of HMW and LMW glutenin subunits}

The gliadin and glutenin subunits were extracted from individually ground grains and the proteins were separated on $10 \%$ polyacrylamide gels in the presence of sodium dodecyl sulphate (SDS-PAGE) (Hailu et al. 2006). Then, in order to determine the gluten composition, the gels were stained with Coomassie Brilliant Blue R-250 solution at-least overnight according to Johansson et al. (1993) and de-stained in $8 \%(\mathrm{w} / \mathrm{v})$ trichloroacetic acid (TCA) for a day. Finally, the nomenclature or designations of Payne and Lawrence (1983) and Nieto-Taladriz et al. (1997) were used for the high molecular weight (HMW) and low molecular weight (LMW) glutenin subunits (GS), respectively.

\section{Preparation of flour}

An IKA A10 experimental mill was used to mill the tetraploid wheat samples at a maximum speed of 20,000 rpm. The whole wheat flour was then used for protein extraction and analysis by High Performance Liquid Chromatography (HPLC).

\section{SE (Size Exclusion)-HPLC}

The SE-HPLC analyses carried out in duplicates on two separately extracted samples, basically following (Labuschagne et al. 2004) method. The two-step protein extraction procedure developed by Gupta et al. (1993) was applied with some modification. For the first extraction step, the proteins soluble in SDS; $11 \mathrm{mg}$ of tetraploid whole wheat flour were suspended in $1.0 \mathrm{ml}$ of $0.5 \%$ (w/v) SDS-phosphate buffer $(\mathrm{pH} 6.9)$ and the samples were vortexed for $10 \mathrm{sec}$. Samples were then stirred for $5 \mathrm{~min}$ at 2,000 rpm and centrifuged for $30 \mathrm{~min}$ at 10,000 rpm to obtain the supernatant. Following centrifugation, the supernatant was filtered through a $0.45 \mu \mathrm{m}$ Acrodisc ${ }^{\circledR}$ non-sterile syringe filter into a glass vial before injecting it into the HPLC system.

For the second extracts that contain proteins soluble in SDS only after sonication, the pellet was subsequently re-suspended in $1.0 \mathrm{ml}$ of $0.5 \%(\mathrm{w} / \mathrm{v}) \mathrm{SDS}$-phosphate buffer and sonicated in an ultrasonic disintegrator fitted with a 3-mm exponential tip, for $30 \mathrm{sec}$ at amplitude 5. The samples were then centrifuged for $30 \mathrm{~min}$ at 10,000 rpm. The supernatants were filtered as before and then injected into the HPLC system. Samples were analyzed in duplicate.

The SE-HPLC analyses were performed on a Shimadzu LC-20AT system, with a UV/ VIS photodiode array detector according to Labuschagne et al. (2004). The percentage of total unextractable polymeric protein in the total polymeric protein was calculated according to Gupta et al. (1993) and Johansson et al. $(2005,2008)$ with modifications.

\section{RP (Reverse Phase)-HPLC}

Protein extraction was carried out in two steps starting with extracting the gliadins in the first step, followed by the glutenins in the second step. The extraction procedure of proteins for RP-HPLC analyses developed by Marchylo et al. (1989) was used with some 
modification according to Labuschagne and Aucamp (2003). In this procedure, the proteins were extracted stepwise to isolate gliadins and glutenin subunits. Hundred milligrams of tetraploid whole wheat flour samples were extracted with $1 \mathrm{ml} \mathrm{70 \%} \mathrm{(v/v)} \mathrm{etha-}$ nol for $30 \mathrm{~min}$ at room temperature with continuous shaking. Following centrifugation at $14,000 \mathrm{rpm}$ for $5 \mathrm{~min}$, the supernatant was filtered through an Acrodisc $\AA 13 \mathrm{ml}$ Syringe filter and was transferred at $4{ }^{\circ} \mathrm{C}$ till analysis.

\section{Statistical analyses}

The SAS software program (SAS, 2004) was used to evaluate differences among varieties and countries of release. Also, the relationships among pheno-quality characters and the composition of grain protein parameters from HPLC data were studied using this program. Spearman rank correlation analyses, ANOVA followed by mean value calculations and separation of means using the Duncan post-hoc analysis as well as principal component analysis (PCA) was applied for the analysis.

\section{Results}

\section{Pheno-quality}

Large variation was obtained among the varieties for all pheno-quality characters evaluated (Table S2). Significant differences were also found between the durum wheat material originating from Ethiopia and that originating from Spain. The material originating from Ethiopia showed longer time to heading, shorter height, darker glumes with less hair, lighter, less dense and longer spikes, and lighter kernels compared to those originating from Spain.

\section{Protein composition}

Table S1 summarizes the allelic composition at the Glu-A1, Glu-B1, Glu-A3, Glu-B3 and Glu-B2 loci of the durum wheat varieties analyzed. At Glu-A1, only one durum wheat, Arendeto, was found with the $2 * * * *$ subunit, while all the other investigated varieties showed the null allele. The most frequent subunits at Glu-B1 were $7+8$ present in $73 \%$ of the varieties, followed by the subunit 20 in $33 \%$ of them. Subunits such as $14+15$ and $6+8$ were also observed in the investigated durum wheat. A total of 13 alleles of LMW glutenin subunits were found in the material, and of these five, six and two alleles were encoded by the Glu-A3, Glu-B3 and Glu-B2 loci, respectively. No obvious difference in composition of HMW glutenin subunits could be detected in the wheat material used in the study. 


\section{Amount and size distribution of polymeric protein}

A significant relationship was found among the released varieties in protein composition measured through SE-HPLC (Table S3). The protein factors measured and evaluated, SDS-extractable and SDS-unextractable PP and MP as well as TOTE, TOTU, \%UPP and $\%$ Large UPP were found to vary significantly with variety (Table S3).

\section{Quantification of protein fraction by RP-HPLC}

A total of 34 gliadin peaks were differentiated by RP-HPLC. Furthermore, a total of 46 glutenin (Glu) peaks were resolved while separating the glutenin extract on the RPHPLC. Presence and absence of peaks varied among the varieties as did the amount of various peaks. A significant positive or negative correlation was also found between some of the specific peaks detected. Low total amounts of gliadins (Gli) were found in the varieties in Boohai and Foka while high amounts were found in Tob-66, and Mexicali. For total amounts of glutenins, low amounts were found in the Ethiopian varieties Quamy and Ude while high amounts were found in Tob-66, Mexicali, LD-357 and Langdon. No overall significant difference in total amounts of gliadins and glutenins were found between Ethiopian and Spanish wheat.

\section{Relationships among pheno-quality data and protein parameters}

Significant positive correlation was found between days to heading (DH) to TOTE and Gli. A significant negative correlation was found between spike density (SD) and both TOTE and Gli. Furthermore, there was a significant negative correlation between TOTE and kernel length (KL) and between Gli and awn length (AL). A negative significant correlation was also found between TOTU and Glu and plant height (PH) (Table S4). A strong significantly positive correlation was found between TOTE and Gli, and TOTU and Glu, respectively. Percentages of UPP and of Large UPP also correlated significantly and positively with some of the traits and peaks as described above.

While evaluating variation of pheno-quality parameters and protein parameters measured by SE-HPLC applying principal component analyses, the first and second principal component was found to explain 25.9 and $25.4 \%$ of the total variation, respectively (Fig. S1A). The first principal component was strongly associated with spike density, awn length, awn colour, days to heading, total extractable protein and plant height. Thus, the first component can be regarded as a yield/grain protein concentration component. The second principal component was associated with total unextractable polymeric protein, glume color, large unextractable polymeric protein in total large polymeric protein, and spike length and thereby had more of a protein polymerisation element in it. The principal component analyses did not group the varities according to the origin (Fig. S1B). Thus, there was a mixture of high and low values of specific quality parameters in both the Ethiopian and the Spanish plant material. For example, the Ethiopian variety Yerer had 
high values of \%UPP and \%Large UPP while the Ethiopian variety Foka had low values of those protein parameters.

Principal component analyses on the RP-HPLC data showed that the first two principal component analyses only explained $39.7 \%$ of the total variation among varieties (data/ figure not shown). Furthermore, the principal component analyses based on RP-HPLC data was not able to differentiate the varieties originating from Spain and Ethiopia.

\section{Discussion}

The present study showed a large variation in amount and size distribution of polymeric proteins as well as in protein composition determined by SE-HPLC, RP-HPLC and SDSPAGE among Ethiopian released tetraploid wheat varieties of durum wheat. The results also showed that the Ethiopian released durum wheat varieties out leveled the Spanish released varieties included in the present study, in terms of variation in amount and size distribution of proteins when grown in similar environmental conditions. Thus, there might be a possibility to breed Ethiopian durum wheat varieties that meet the quality requirements for African as well of European pasta production industry.

The Ethiopian released varieties showed some variation in composition of HMW-GS although all of the varieties showed either $6+8,7+8$ or 20 or a mixture of these. One variety was found showing the $2 * * * *$ subunit, previously reported in Ethiopian durum wheat by (Hailu et al. 2006). A larger variation was seen in the composition of LMW-GS, which also corresponds to previous investigations (Hailu et al. 2006). Specific protein composition in durum wheat has been related to quality for pasta production, e.g. LMW2 and LMW-1 have been related to good and poor pasta quality, respectively (Pogna et al. 1990; Ruiz and Carrillo 1995; Labuschagne et al. 2004; Martinez et al. 2004). Furthermore Glu-A3a and Glu-A3d have been correlated to good pasta quality (Branlard et al. 2003). Equivalence models for allelic composition, LMW patterns and quality have been developed (Payne et al. 1984; Nieto-Taladriz et al. 1997) and these models have also been adapted to the Ethiopian durum wheat material (Hailu et al. 2006). Applying this model indicate a good quality as related to LMW-GS of several of the Ethiopian varieties, i.e. Arendeto, Boohai, Foka, Klinto, LD-357, Tob-66, UDE and Yerer. However, some of the mentioned varieties were not found to be homogeneous which can influence quality performances of the material. Some of the mentioned varieties showed HMW-GS 20 encoded at Glu-B1 (Boohai, Foka, Yerer), known to be related to less gluten strength than, e.g. HMW-GS $7+8$ (Johansson and Svensson 1995). Regarding the durum wheat of Spanish origin, it showed similar diversity as the Ethiopian durum wheat in terms of HMW- and LMW-GS.

RP-HPLC analyses also showed large variation among varieties in composition of protein subunits with specific peaks in certain varieties and also with variations of amounts of certain peaks. Almost no significant differences in amounts of specific peaks were however found in the Ethiopian wheat as related to the Spanish wheat. Thus, the RP-HPLC data mainly revealed the diversity of the material investigated although specific peaks could not be related to quality data. As for total amounts of gliadins and glu- 
tenins variation was found among the varieties with Tob-66 and Mexicali both having high total gliadins and glutenins indicating high protein concentration in these varieties. The varieties LD-357 and Langdon showed high total glutenins with no corresponding high gliadins which might indicate high gluten strength in these varieties. High total glutenin/gliadin content has in several investigations been reported to correspond to high gluten strength (Sissons et al. 2005).

The TOTE has in previous investigation been used as an indicator of grain protein concentration (Malik et al. 2011, 2013). A large variation was found for TOTE in the present investigation, with high values in the Ethiopian varieties Tob-66, Quamy and Foka while low values were found in the Ethiopian varieties Boohai and LD-357, and the Spanish variety Alaga. The lowest value was only $56 \%$ of the highest value indicating large variation in protein concentration in the material. Previous investigations have shown a clear positive correlation between protein concentration and bread volume (Finney and Barmore 1948), but also with protein concentration and decreased gluten strength and increased relative amount of gliadins to glutenins in the flour (Johansson et al. 2013). Thus, protein concentration in the durum wheat grain contributes to pasta quality where the protein network during the drying process is known to be an important quality parameter (Finney and Barmore 1948). While comparing the Ethiopian varieties with the Spanish varieties, no general significant relationship due to origin of the wheat was found.

The percentage of UPP has in previous investigations been related to gluten strength in wheat (Malik et al. 2011, 2013) although \%UPP during mixing has also been found to change independently as related to content in the mature grain (Hussain et al. 2012). The latter is thus an important character, as changes of UPP and formation of a suitable network during the drying process is important features to produce pasta of good quality (Bruneel et al. 2010; Johansson et al. 2013). A large variation was found in \%UPP in the durum wheat varieties in the present investigation, with high values in Yerer, Mexicali, Langdon and LD-357, and low values in Foka, Quamy and Ude. The lowest value was only $40 \%$ of the highest value indicating large variation in the gluten strength of the material.

The present study showed a highly significant correlation between TOTE and the total amount of gliadins as well as between TOTU and \%UPP and the total amount of glutenin. These results correspond to a significant correlation between the grain protein concentration, total gliadin content and TOTE that has been revealed in several previous studies (Carrillo et al. 1990). The relationship between total amount of glutenins, extractability of proteins and polymerization of the gluten proteins has also previously been claimed (Carrillo et al. 2000) although maybe not as clearly shown as in the present study.

One aim of the present study was to evaluate whether any significant relationships existed among the evaluated protein parameters and the different quality parameters used for quality assessment of the Ethiopian durum wheat varieties. The most interesting correlation data obtained is the positive correlation between TOTE and total amount of gliadins and days to heading meaning that the later the variety matured the higher grain protein concentration the variety obtained. A later maturation time of wheat is often related 
to higher green biomass accumulation during plant development and thereby to a higher grain starch accumulation during grain filling (Johansson et al. 2013). A higher starch accumulation in the grain is well known to lead to lower protein concentration in the grain as the protein accumulation does not increase to the same extent as do the starch accumulation (Jenner and Rathjen 1972). Thus, the positive correlation between grain protein concentration and days to heading in the present study was not expected and must be explained by the use of diverse genetic material. The most likely explanation for the found correlations is genetically determined variation in grain protein concentration and maturation time and we accidentally must have selected some varieties performing different than the ones usually found. Another possible explanation might be that most relationships previously reported refer to hexaploid bread wheat grown under European or the USA growing conditions and the present study was carried out on durum wheat grown in Ethiopia. Thus, more studies are needed applying growing conditions from other areas than the ones in Europe and the USA. The seed texture parameter evaluated in the present study can be seen as related to grain hardiness, an important parameter for pasta production (Sissons et al. 2005). Highest values for seed texture were seen for the varieties Arendeto, Asassa, Klinto, Yerer and the Spanish variety Alaga. No significant differences were seen between the wheat of different origin. The yellow color of the flour is also an important character for pasta production, a large number of the evaluated varieties showed yellow kernels, Asassa, CDB and LD-357 showed white kernels.

A large variation was seen in the investigated Ethiopian released durum wheat varieties regarding protein composition, amount and size distribution of polymeric proteins and pheno-quality data. The protein evaluations here indicated some of the Ethiopian varieties to be better for pasta production than others. Equivalence modeling of the allelic composition of the Ethiopian varieties together with evaluation of HMW-GS indicated that the varieties Klinto, LD-357, Tob-66 and UDE might be interesting for pasta production (part of Arendeto and Boohai as well although these two varieties were not homozygous). High values of protein parameters indicating gluten strength (high total glutenin content, \%UPP) was found in the varieties Tob-66 (high Glu), LD-357 (high Glu and $\%$ UPP) and Yerer (high \%UPP). Tob-66 also showed high total gliadin content and high TOTE, thus having high protein concentration.

\section{Acknowledgements}

The laboratory work was supported by UD-40 project (an initiative from the Ministry of Foreign Affairs in Sweden administered through the Swedish University of Agricultural Sciences), the final write up was supported by Swedish Institute (SI) and plant materials were obtained from Debre Zeit Agricultural Research Centre of Ethiopia and Dr NietoTaladriz for which we are very grateful. 


\section{References}

Bechere, E., Peña, R.J., Mitiku, D. 2002. Gluten composition, quality characteristics, and agronomic attributes of durum wheat cultivars released in Ethiopia. Afric. Crop Sci. J. 10:173-182.

Boggini, C., Pogna, N.E. 1989. The bread making quality and storage protein composition of Italian durum wheat. J. Cereal Sci. 9:131-138.

Branlard, G., Dardevet, M., Amiour, N., Igrejas, G. 2003. Allelic diversity of HMW and LMW glutenin subunits and omega gliadins in French bread wheat (Triticum aestivum L.). Genet. Resou. Crop Evol. 50:669679.

Bruneel, C., Pareyt, B., Brijs, K., Delcour, J.A. 2010. The impact of the protein network on the pasting and cooking properties of dry pasta products. Food Chem. 120:371-378.

Carrillo, J.M., Martinez, M.C., Moita, C., Brites, M.T., Nieto Taladriz, M.T., Vazquez, J.F. 2000. Relationship between endosperm proteins and quality in durum wheat (Triticum turgidum L. var. durum). Options Mediterranees 40:463-467.

Carrillo, J.M., Vazquez, J.F., Orellana, J. 1990. Relationship between gluten strength and glutenin proteins in durum wheat cultivars. Plant Breed. 104:325-333.

Ciaffi, M., Tozzi, L., Lafiandra, D. 1996. Relationship between flour protein compositions determined by sizeexclusion high-performance liquid chromatography and dough rheological parameters. Cereal Chem. 73:346-351.

El Haddad, L., Aussenac, T., Fabre, J.L., Sarrafi, A. 1995. Relationships between polymeric glutenin and the quality characteristics for seven common wheats (Triticum aestivum) grown in the field and greenhouse. Cereal Chem. 72:598-601.

Finney, K.F., Barmore, M.A. 1948. Loaf volume and protein content of hard winter and spring wheats. Cereal Chem. 25:291-312.

Fu, B.X., Sapirstein, H.D. 1996. Procedure for isolating monomeric proteins and polymeric glutenin of wheat flour. Cereal Chem. 73:143-152.

Gulia, N., Khatkar, B.S. 2015. Quantitative and qualitative assessment of wheat gluten proteins and their contribution to instant noodle quality. Int. J. Food Properties 18:1648-1663.

Gupta, R.B., Khan, K., Macritchie, F. 1993. Biochemical basis of flour properties in bread wheats. I. Effects of variation in quantity and size distribution of polymeric protein. J. Cereal Sci. 18:23-41.

Gupta, R.B., Macritchie, F. 1994. Allelic variation at glutenin subunit and gliadin loci, Glu-1, Glu-3 and Gli-1 of common wheats. II. Biochemical basis of the allelic effects on dough properties. J. Cereal Sci. 19:19-29.

Gupta, R.B., Popineau, Y., Lefebvre, J., Cornec, M., Lawrence, G.J., MacRitchie, F. 1995. Biochemical basis of flour properties in bread wheats. II. Changes in polymeric protein formation and dough/gluten properties associated with the loss of low Mr or high Mr glutenin subunits. J. Cereal Sci. 21:103-116.

Gupta, R.B., Masci, S., Lafiandra, D., Bariana, H.S., MacRitchie, F. 1996. Accumulation of protein subunits and their polymers in developing grains of hexaploid wheats. J. Exp. Bot. 47:1377-1385.

Hailu, F., Johansson, E., Merker, A., Belay, G., Harjit-Singh, Zeleke, H. 2006. Composition of and variation in high- and low molecular weight glutenin subunits, and omega gliadins in Ethiopian tetraploid wheat germplasm. Plant Genetic Resour. 4:134-143.

Hailu, F., Johansson, E., Merker, A. 2010. Patterns of phenotypic diversity for phonologic and qualitative traits in Ethiopian tetraploid wheat germplasm. Genet. Res. Crop Evol. 57:781-790.

Hussain, A., Larsson, H., Kuktaite, R., Prieto-Linde, M.L., Johansson, E. 2012. Towards the understanding of bread-making quality in organically grown wheat: dough mixing behavior, protein polymerization and structural properties. J. Cereal Sci. 56:659-666.

Jenner, C.F., Rathjen, A.J. 1972. Limitations to the accumulation of starch in the developing wheat grain. Ann. Bot. 36:743-754.

Johansson, E., Henriksson, P., Svensson, G., Hennen, W.K. 1993. Detection, chromosomal location and evaluation of the functional value of a novel high Mr glutenin subunit found in Swedish wheats. J. Cereal Sci. 17:237-245.

Johansson, E., Svensson, G. 1995. Contribution of the high-molecular-weight subunit $21 *$ to bread making quality of Swedish wheats. Cereal Chem. 72:287-290. 
Johansson, E., Kuktaite, R., Andersson, A., Prieto-Linde, M.L. 2005. Protein polymer built-up during wheat development: influences of temperature and nitrogen timing. J. Sci. Food Agric. 85:473-479.

Johansson, E., Prieto-Linde, M.L., Gissen, C. 2008. Influences of weather, cultivar and fertiliser rate on grain protein polymer accumulation in field-grown winter wheat, and relations to grain water content and falling number. J. Sci. Food Agric. 88:2011-2018.

Johansson, E., Malik, A.H., Hussain, A., Rasheed, F., Newson, W.R., Plivelic, T., Hedenqvist, M.S., Gällstedt, M., Kuktaite, R. 2013. Wheat gluten polymer structures: The impact of genotype, environment and processing on their functionality in various applications. Cereal Chem. 90:367-376.

Labuschagne, M.T., Aucamp, U. 2003. The use of size exclusion high performance liquid chromatography (SE-HPLC) for wheat quality prediction in South Africa. South African J. Plant Soil 21:8-12.

Labuschagne, M.T., Koen, E., Dessalegn, T. 2004. Use of size-exclusion high-performance liquid chromatography for wheat quality prediction in Ethiopia. Cereal Chem. 81:533-537.

Liu, C.Y., Shepherd, K.W., Rathjen, A.J. 1996. Improvement of durum wheat pasta making and bread making qualities. Cereal Chem. 73:155-166.

Malik, A.H., Prieto-Linde, M.L., Kuktaite, R., Andersson, A., Johansson, E. 2011. Individual and interactive effects of genetic background and environmental conditions on amount and size distribution of polymeric proteins in wheat grain. Czech J. Genet. Plant Breed. 47:5186-5189.

Malik, A.H., Kuktaite, R., Johansson, E. 2013. Combined effect of genetic and environmental factors on the accumulation of proteins in the wheat grain and their relationship to bread-making quality. J. Cereal Sci. 57:170-174.

Martinez, M.C., Ruiz, M., Carrillo, J.M. 2004. New B low Mr glutenin subunit alleles at the Glu-A3, Glu-B2 and Glu B3 loci and their relationship with gluten strength in durum wheat. J. Cereal Sci. 40:101-107.

Marchylo, B.A., Kruger, J.E., Hatcher, D.W. 1989. Quantitative reversed phase high-performance liquid chromatographic analysis of wheat storage proteins as a potential quality prediction tool. J. Cereal Sci. 9:113130.

Nieto-Taladriz, M.T., Ruiz, M., Martinez, M.C., Vazquez, J.F., Carrillo, J.M. 1997. Variation and classification of B low-molecular weight glutenin subunit alleles in durum wheat. Theor. Appl. Genet. 95:1115-1160.

Payne, P.I., Lawrence, G.J. 1983. Catalogue of alleles for the complex gene loci, Glu-A1, Glu-B1 and Glu-D1, which code for the high-molecular-weight subunit of glutenin in hexaploid wheat. Cereal Res. Commun. 11:29-35.

Payne, P.I., Jackson, E.A., Holt, L.M. 1984. The association between $\gamma$-gliadin 45 and gluten strength in durum wheat varieties: a direct causal effect or the result of genetic link-age. J. Cereal Sci. 2:73-81.

Peña, R.J., Trethowan, R.M., Pfeiffer, W.H., van Ginkel, M. 2002. Quality (end-use) improvement in wheat, compositional, genetic, and environmental factors. In: Basra, A.S., Randhawa, L.S. (eds), Quality Improvement in Field Crops. Food Product Press. New York, USA. pp. 1-37.

Pogna, N.E., Autran, J.C., Mellini, F., Lafiandra, D., Feillet, P. 1990. Chromosome 1B-encoded gliadins and glutenin subunits in durum wheat: genetics and relationship to gluten strength. J. Cereal Sci. 11:15-34.

Rharrabti, Y., Villegas, D., Royo, C., Martos-Nũñez, V., García del Moral, L.F. 2003. Durum wheat quality in Mediterranean environments II. Influence of climatic variables and relationships between quality parameters. Field Crops Res. 80:133-140.

Ruiz, M., Carrillo, J.M. 1995. Relationships between different prolamin proteins and some quality parameters in durum wheat. Plant Breeding 114:40-44.

SAS 2004. SAS/STAT TM Users guide. SAS Institute Inc., Cary, NC, USA.

Sissons, M.J., Ames, N.P., Hare, R.A., Clarke, J.M. 2005. Relationship between glutenin subunit composition and gluten strength measurements in durum wheat. J. Sci. Food Agric. 85:2445-2452.

Wieser, H., Antes, S., Seilmeier, W. 1998. Quantitative determination of gluten protein types in wheat flour by reversed-phase high-performance liquid chromatography. Cereal Chem. 75:644-650. 


\section{Electronic Supplementary Material (ESM)}

Electronic Supplementary Material (ESM) associated with this article can be found at the website of CRC at http://www.akademiai.com/content/120427/

Electronic Supplementary Table S1. Plant material used for the study and their composition of gluten subunits encoded from various loci at chromosomes $\mathrm{A}_{1}, \mathrm{~A}_{3}, \mathrm{~B}_{1}-\mathrm{B}_{3}$

Electronic Supplementary Table S2. Mean values of measured pheno-quality traits and protein content in 15 different durum wheat varieties

Electronic Supplementary Table S3. Mean square from ANOVA of extractable and unextractable proteins obtained from peak areas of chromatograms

Electronic Supplementary Table S4. Spearman rank correlation coefficients between protein parameters and different quality parameters of flour for fifteen tetraploid wheat varieties

Figure S1. Loading (A) and score plot (B) from principal component analysis of pheno-quality data and protein parameters from SE-HPLC in released varieties of durum wheat of Ethiopian and Spanish origin. Days to heading $(\mathrm{DH})$, days to maturity $(\mathrm{DM})$, plant height $(\mathrm{PH})$, glume colour $(\mathrm{GC})$, glume hairiness $(\mathrm{GH})$, awn colour $(\mathrm{AC})$, awn length (AL), spike density (SD), spike length (SL), beak shape (BS), kernel length (KL), kernel colour (KC), seed texture (SN), Total SDS-extractable protein (TOTE), Total SDS-Unextractable Protein (TOTU), Percent of Large Unextractable Polymeric Protein in the total large polymeric protein (LUPP), Percent of Unextractable Polymeric Protein in the total polymeric protein (TUPP) 